\title{
Aprendizagem móvel e ubíqua em Odontologia: revisão integrativa da literatura
}

\author{
Mobile and ubiquitous learning in Dentistry: integrative literature review \\ Aprendizaje móvil y ubicuo en Odontología: revisión integradora de la literatura
}

\author{
Isadora Morgana Caixeta \\ ORCID: https://orcid.org/0000-0002-7316-5768 \\ Centro Universitário de Patos de Minas, Brasil \\ E-mail: isadoracaixeta@unipam.edu.br \\ Maria Teresa Borges Araújo \\ ORCID: https://orcid.org/0000-0002-6866-1345 \\ Centro Universitário de Patos de Minas, Brasil \\ E-mail: mariateresa@unipam.edu.br \\ Yuri Kayano Souza Guimarães \\ ORCID: https://orcid.org/0000-0002-3870-3962 \\ Centro Universitário de Patos de Minas, Brasil \\ E-mail:yuriksg@unipam.edu.br \\ Cássia Eneida de Souza Vieira Dutra \\ ORCID: https://orcid.org/0000-0002-9036-6203 \\ Centro Universitário de Patos de Minas, Brasil \\ E-mail: cassiaesv@unipam.edu.br \\ Fabrício Campos Machado \\ ORCID: https://orcid.org/0000-0003-4603-8795 \\ Centro Universitário de Patos de Minas, Brasil \\ E-mail: fabriciocampos@unipam.edu.br \\ Thiago de Amorim Carvalho \\ ORCID: https://orcid.org/0000-0003-1153-0931 \\ Centro Universitário de Patos de Minas, Brasil \\ E-mail: thiagocarvalho@unipam.edu.br
}

\begin{abstract}
Resumo
Com a pandemia da COVID-19, e o distanciamento social imposto por ela houve necessidade de se elaborar estratégias para que o processo ensino-aprendizagem fosse mantido. Dessa forma conceitos como aprendizagem ubíqua e aprendizagem móvel passaram a figurar entre estudantes, professores e toda a comunidade acadêmica, inclusive nos cursos de Odontologia. Sendo assim a compreensão sobre essas formas não tradicionais de aprendizagem se torna necessária, no intuito de que sua aplicação seja feita de maneira responsável. O objetivo deste trabalho foi caracterizar a aprendizagem móvel e ubíqua e suas aplicações em Odontologia. Foi realizada uma revisão integrativa da literatura realizada por meio das bases PubMed e Scielo. A estratégia de busca considerou os termos:" aprendizagem móvel e odontologia" "aprendizagem ubíqua e odontologia" e seus correspondentes em inglês "mobile learning and dentistry"” ubiquitous learning and dentistry", em um período de 10 anos (2011 a 2021). A análise descritiva, à luz da literatura científica nacional e internacional, permitiu agrupar uma amostra de oito artigos com evidências científicas de maneira a caracterizar a aprendizagem móvel e ubíqua e suas aplicações em Odontologia, identificar quais as ferramentas utilizadas para esse fim e seu impacto no processo ensino-aprendizagem. A partir do exposto percebe-se que a nova realidade imposta e acelerada pela pandemia da COVID-19, em relação ao uso das ferramentas digitais de ensino-aprendizagem, especialmente a aprendizagem móvel e ubíqua, é possível e já se mostra eficaz principalmente quando associada aos encontros presenciais, embora mais estudos sejam necessários para verificar seus impactos diretos sobre estudantes e docentes.
\end{abstract}

Palavras-chave: Educação online; Aprendizado online; Ensino; Odontologia.

\begin{abstract}
With the pandemic of COVID-19, and the social distancing imposed by it, there was the need to develop strategies for the teaching-learning process to be maintained. Thus, concepts such as ubiquitous learning and mobile learning have started to appear among students, professors, and the entire academic community, including dentistry courses. Thus, the understanding of these non-traditional forms of learning becomes necessary, so that their application can be done in a responsible way. The objective of this work was to characterize mobile and ubiquitous learning and its applications in dentistry. An integrative literature review was conducted using the PubMed and Scielo databases. The search strategy considered the terms: "mobile learning and dentistry" "ubiquitous learning and dentistry" and their corresponding terms in English "mobile learning and dentistry", over a period of 10 years (2011 to 2021). The descriptive analysis, in the light of the national and international scientific literature, allowed grouping a sample of
\end{abstract}


eight articles with scientific evidence to characterize mobile and ubiquitous learning and its applications in dentistry, identify which tools are used for this purpose and their impact on the teaching-learning process. Based on the above, the new reality imposed and accelerated by the COVID-19 pandemic, regarding the use of digital teaching-learning tools, especially mobile and ubiquitous learning, is possible and has already proven to be effective, especially when associated with face-to-face meetings, although further studies are needed to verify its direct impacts on students and teachers.

Keywords: E-learning; Online learning; Teaching; Dentistry.

\begin{abstract}
Resumen
Con la pandemia del COVID-19, y el distanciamiento social que impuso, fue necesario elaborar estrategias para que el proceso de enseñanza-aprendizaje pudiera mantenerse. Así, conceptos como aprendizaje ubicuo y aprendizaje móvil empezaron a aparecer entre los estudiantes, los profesores y toda la comunidad académica, incluso en los cursos de odontología. Así, la comprensión de estas formas no tradicionales de aprendizaje se hace necesaria para aplicarlas de forma responsable. El objetivo de este trabajo fue caracterizar el aprendizaje móvil y ubicuo y sus aplicaciones en Odontología. Se realizó una revisión bibliográfica integradora utilizando las bases de datos PubMed y Scielo. La estrategia de búsqueda consideró los términos: "mobile learning and dentistry", "ubiquitous learning and dentistry" y sus correspondientes en inglés: "mobile learning and dentistry", "ubiquitous learning and dentistry", durante un periodo de 10 años (2011 a 2021). El análisis descriptivo, a la luz de la literatura científica nacional e internacional, permitió agrupar una muestra de ocho artículos con evidencia científica para caracterizar el aprendizaje móvil y ubicuo y sus aplicaciones en Odontología, identificar qué herramientas se utilizan para este fin y su impacto en el proceso de enseñanza-aprendizaje. A partir de lo expuesto, se puede notar que la nueva realidad impuesta y acelerada por la pandemia del COVID-19, en relación con el uso de herramientas digitales de enseñanza-aprendizaje, especialmente el aprendizaje móvil y ubicuo, es posible y ya es efectiva, principalmente cuando se asocia a los encuentros presenciales, aunque son necesarios más estudios para verificar sus impactos directos en los alumnos y profesores.
\end{abstract}

Palabras clave: Educación a distancia; Aprendizaje online; Enseñanza; Odontología.

\title{
1. Introdução
}

A educação digital é uma ampla construção que descreve uma série de estratégias de ensino e aprendizagem baseadas no uso de mídias eletrônicas e dispositivos como ferramentas de treinamento, comunicação e interação. O instrutivo abrange aspectos que incluem abordagens educacionais, conceitos, métodos e tecnologias. A educação digital descomplica a aprendizagem remota, o que pode beneficiar educadores e alunos, reduzindo assim, tempo e barreiras geográficas a formação (Leite, 2018; Dunleavy, et al. 2019; Silva, et al. 2021).

O conceito de aprendizagem ubíqua definido por Santaella (2010) é dado como a aprendizagem que pode se dar por diversas ferramentas digitais, em qualquer espaço físico e temporal. Nos dias atuais, é comum se observar o uso da tecnologia no processo ensino aprendizagem. A era da tecnologia provocou mudanças significativas no âmbito educacional, permitido o surgimento de novas alternativas com relação aos recursos educacionais. Com o crescente avanço da tecnologia, o desenvolvimento das redes de dados, acesso à internet, permitiu a conexão com diversos dispositivos, que vem contribuindo com o acesso à conteúdos educacionais a qualquer momento, e em qualquer lugar. Diante disso, surgiu-se então o novo modelo de ensino a distância chamado de "aprendizagem móvel”( Elkheir \& Mutabil, 2015; Sales \& Boscarioli, 2020).

Também conhecida como m-learning, a aprendizagem móvel, surgiu como uma alternativa de modo de aprendizagem, no qual o estudante tem a oportunidade de aprender através de diversos contextos e várias interações entre dispositivos. Se trata de:

uma etapa renovada do aprendizado mediado pela tecnologia, em que os alunos têm acesso as informações, e podem optar por aprender individualmente ou em equipes, dentro ou fora da sala de aula convencional, usufruindo de seus dispositivos móveis pessoais como ferramenta de aprendizagem (Hinojo-Lucena, et al. 2019; Ballesteros, et al. 2020; Vieira \& Silva, 2020).

Trata-se, portanto, de uma concepção tecnológica que proporciona um universo imenso de métodos de ensino e enriquece o trabalho pedagógico centrado no estudante. Entretanto, é um método que demanda desse maior interesse, 
concentração e comprometimento, e por desviar do método de ensino convencional, a adaptação a este modelo nem sempre é alcançada de maneira satisfatória (Arce \& Pegueros, 2017; Bernardete, 2020). O ensino prático voltado para as disciplinas clínicas ainda se apresenta em fase de retomada com as adequações necessárias, principalmente do ponto de vista da biossegurança. Para as disciplinas teóricas melhorias devem ser implementadas no que se diz respeito à metodologias de ensino e práticas pedagógicas, preparo dos estudantes e também de docentes para usufruírem das possibilidades do ensino remoto (Medeiros et al., 2021).

O objetivo deste trabalho foi caracterizar a aprendizagem móvel e ubíqua em Odontologia identificando as vantagens oferecidas pela aprendizagem em ambiente digital.

\section{Metodologia}

Trata-se de uma revisão integrativa da literatura com pergunta do estudo de natureza qualitativa elaborada de acordo com a estratégia PICO (Estrela, 2018). Para P (pessoas, pacientes, grupo) atribuiu-se os estudantes de Odontologia, para o I (intervenção) a aplicação da aprendizagem móvel e ubíqua em disciplinas do curso de odontologia para C (controle) uso de métodos tradicionais de ensino-aprendizagem e $\mathrm{O}$ (desfecho) uso de ferramentas digitais para aprendizagem ubíqua e móvel por alunos e professores de Odontologia, impactos no aprendizado de estudantes de Odontologia por meio de ferramentas digitais ubíquas e móveis.

Desta maneira a pergunta do estudo foi a seguinte: é possível utilizar aprendizagem ubíqua e móvel no processo ensino-aprendizagem nas disciplinas do curso de Odontologia?

A busca dos trabalhos foi realizada nas bases de dados: SCIELO (Scientific Electronic Library Online), PUBMED (National Library of Medicine), considerando os seguintes termos chave para a estratégia de busca combinado com o operador booleano "AND”- "aprendizagem móvel e odontologia" "aprendizagem ubíqua e odontologia" e seus correspondentes em inglês "mobile learning and dentistry" "ubiquitous learning and dentistry".A seleção dos artigos foi realizada baseada nos parâmetros de elegibilidade propostos pelo guia PRISMA-Scr.

Como critérios de inclusão foram selecionados artigos dos últimos 10 anos (2010 a 2020), dos idiomas da língua portuguesa e inglesa, disponíveis em texto completo, e cuja metodologia ou desfechos estivessem em consonância com o escopo deste estudo. Foram excluídos da pesquisa artigos de outros idiomas, artigos apresentados em forma de resumos, resumos expandidos, opiniões de especialistas, e artigos que não possuem texto completo disponível na íntegra, além da literatura cinzenta composta por teses, dissertações, monografias, trabalhos de conclusão de curso que embora disponíveis nas bases de dados não passam por processo de revisão pelos pares.

\section{Resultados e Discussão}

A busca nas bases de dados resultou na identificação de 158 artigos potencialmente elegíveis, sendo 70 deles encontrados no PUBMED e 88 artigos encontrados na Scielo. Não foram utilizadas outras fontes de publicações. O processo de seleção e elegibilidade dos artigos está demonstrado na Figura 1.

Com relação ao número total de artigos, foram eliminados 3 duplicados, e a partir de então foi realizada a leitura dos títulos das publicações, eliminando assim 120 artigos. Em seguida foi realizada a leitura de 35 resumos dos artigos selecionados, e mais 15 artigos foram excluídos, após a leitura do resumo, 20 artigos foram selecionados para leitura do texto completo na integra, excluindo aqueles que eram divergentes com o objetivo do estudo e critérios de inclusão e exclusão. 
Figura 1. Fluxograma do processo de seleção dos estudos primários adaptado do Preferred Reporting Items for Systematic review and Meta-Analyses (PRISMA-SCr).

Identificação

Filtro: 10 anos

Seleção

Elegibilidade

Inclusão
Registro identificado no banco de dados de busca (Pubmed - Ubiquitous learning and dentistry $\mathrm{n}=13$

Mobile learning and dentistry $=57$ )

(Scielo aprendizagem móvel e odontologia $\mathrm{n}=81$

Aprendizagem ubíqua e odontologia $\mathrm{n}=7$ )

Registro após eliminar os duplicados $(\mathrm{n}=155)$

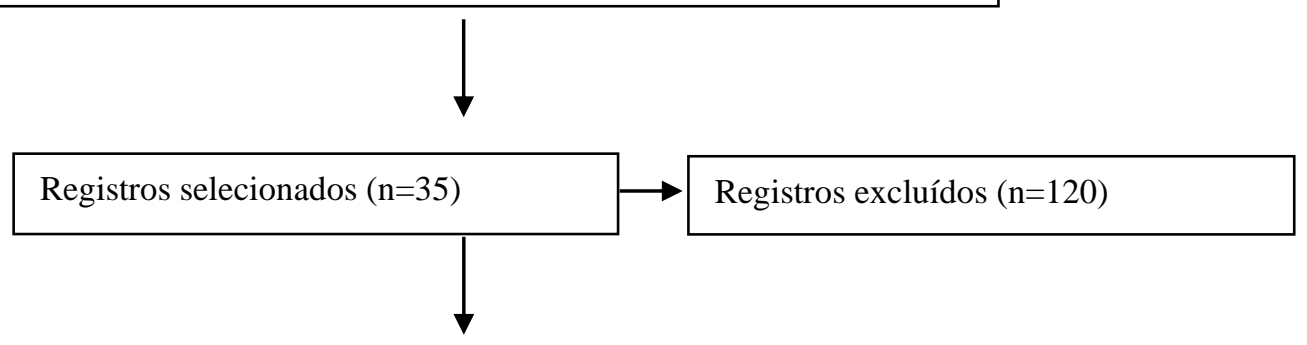

Artigo em texto completo avaliados para elegibilidade $(\mathrm{n}=20)$

Estudos incluídos em síntese qualitativa $(\mathrm{n}=8)$

Fonte: Autores (2021).

Após a leitura das publicações avaliadas para elegibilidade, alcançou-se então, uma amostra final de oito estudos, que após a leitura do texto completo na integra, os dados foram organizados para análise e interpretação das pesquisadoras. Os estudos foram analisados quanto a qualidade metodológica através do instrumento Mixed Methods Appraisal Tool (MAAT), aplicado por duas pesquisadoras independentes.

A extração dos dados dos estudos selecionados ocorreu a partir da utilização do instrumento validado por Ursi (2005) que permitiu a organização dos resultados em um quadro-síntese contendo as seguintes informações: autor(es), ano de publicação, periódico, tipo de estudo, objetivos do estudo, metodologia, resultados e nível de evidência. A análise descritiva, à luz da literatura científica nacional e internacional, permitiu agrupar as evidências de maneira a caracterizar a aprendizagem móvel e ubíqua e suas aplicações em Odontologia, identificar quais as ferramentas utilizadas para esse fim e seu impacto no processo ensino-aprendizagem. O quadro 1 apresenta a caracterização dos estudos selecionados para a análise qualitativa. 
Quadro 1. Caracterização dos artigos selecionados de acordo com autor, ano de publicação, objetivo, tipo de estudo e resultados principais.

\begin{tabular}{|c|c|c|c|c|}
\hline $\begin{array}{c}\text { Autor e ano de } \\
\text { publicação }\end{array}$ & Periódico & Objetivo & Tipo de estudo & Resultados \\
\hline $\begin{array}{l}\text { Rung, Warnke, \& } \\
\text { Mattheos, 2014. }\end{array}$ & $\begin{array}{c}\text { JMIR mHealth e } \\
\text { uHealth }\end{array}$ & $\begin{array}{l}\text { Obter uma visão a respeito do uso de } \\
\text { smartphones pelos alunos, e suas } \\
\text { atitudes em relação ao uso educacional } \\
\text { de dispositivos móveis. }\end{array}$ & $\begin{array}{c}\text { Pesquisa } \\
\text { Exploratória }\end{array}$ & $\begin{array}{l}\text { Os resultados deste estudo corroboram que os } \\
\text { alunos usam smartphones e mídias sociais para } \\
\text { suas atividades de aprendizagem, e percebem seus } \\
\text { smartphones como ferramentas de aprendizagem. }\end{array}$ \\
\hline Kon, et al. 2016 & $\begin{array}{l}\text { Wiley online } \\
\text { library }\end{array}$ & $\begin{array}{l}\text { Esclarecer as diferenças entre os } \\
\text { usuários da combinação PC / } \\
\text { dispositivo móvel e do usuário } \\
\text { somente PC em relação à frequência de } \\
\text { acesso e tempo gasto no site pré- } \\
\text { clínico de prótese total. }\end{array}$ & Estudo piloto & $\begin{array}{l}\text { Os resultados implicam que oferecer fácil } \\
\text { acessibilidade ao sistema de e-learning por meio } \\
\text { de dispositivos móveis aumenta as oportunidades } \\
\text { de aprendizagem. }\end{array}$ \\
\hline $\begin{array}{l}\text { Deshpande, } \\
\text { Chahande, \& Rathi, } \\
2017 .\end{array}$ & $\begin{array}{c}\text { Wolters Kluwer } \\
\text { - Medknow }\end{array}$ & $\begin{array}{l}\text { Descrever um aplicativo móvel para } \\
\text { ensinar prótese dentária e avaliar seu } \\
\text { efeito nas tomadas de decisões } \\
\text { clínicas. }\end{array}$ & $\begin{array}{c}\text { Pesquisa } \\
\text { exploratória }\end{array}$ & $\begin{array}{l}\text { Os resultados sugerem que aplicativo de } \\
\text { aprendizagem móvel é uma técnica de ensino útil } \\
\text { para fornecer treinamento para o planejamento da } \\
\text { reabilitação protética para acadêmicos. }\end{array}$ \\
\hline $\begin{array}{l}\text { Mergany, Dafalla, } \\
\& \text { Awooda, } 2021 .\end{array}$ & $\begin{array}{l}\text { BMC Medical } \\
\text { Education }\end{array}$ & $\begin{array}{l}\text { Investigar em que medida o uso de } \\
\text { aprendizagem móvel como um } \\
\text { complemento para aulas clássicas em } \\
\text { sala de aula afeta os alunos }\end{array}$ & $\begin{array}{c}\text { Pesquisa } \\
\text { exploratória }\end{array}$ & $\begin{array}{l}\text { O resultado do estudo indica que maioria dos } \\
\text { participantes apresentou atitude positiva em } \\
\text { relação à eficácia do mobile learning. }\end{array}$ \\
\hline Stamm, et al.2019. & $\begin{array}{l}\text { Head \& Face } \\
\text { Medicine }\end{array}$ & $\begin{array}{l}\text { Avaliar o impacto educacional de um } \\
\text { programa individual de tablet PC } \\
\text { (TPC), analisando estudantes } \\
\text { universitários de Odontologia na } \\
\text { Alemanha. }\end{array}$ & $\begin{array}{c}\text { Revisão integrativa } \\
\text { da literatura }\end{array}$ & $\begin{array}{l}\text { Os resultados obtidos que o TPC tem um efeito } \\
\text { positivo de aprendizagem sobre o conhecimento } \\
\text { teórico em Ortodontia. }\end{array}$ \\
\hline $\begin{array}{c}\text { Suner, Yilmaz \& } \\
\text { Kin } 2019 .\end{array}$ & Peerj journals & $\begin{array}{l}\text { Avaliar hábitos de uso, atitudes e } \\
\text { percepções em relação ao mobile } \\
\text { learning por estudantes de graduação } \\
\text { em odontologia }\end{array}$ & $\begin{array}{c}\text { Pesquisa } \\
\text { exploratória }\end{array}$ & $\begin{array}{l}\text { Os resultados sugerem que estudantes de } \\
\text { odontologia geralmente têm atitudes positivas em } \\
\text { relação ao m-learning. Aplicativos móveis podem } \\
\text { aumentar o desempenho dos alunos. }\end{array}$ \\
\hline $\begin{array}{l}\text { Golshah, et al. } \\
2020 .\end{array}$ & $\begin{array}{l}\text { BMC Medical } \\
\text { Education }\end{array}$ & $\begin{array}{l}\text { Comparar a eficácia do aprendizado } \\
\text { móvel baseado em smartphone versus } \\
\text { o aprendizado baseado em palestras } \\
\text { para a instrução de identificação de } \\
\text { pontos cefalométricos }\end{array}$ & $\begin{array}{l}\text { Estudo } \\
\text { intervencionista } \\
\text { quase experimental } \\
\text { - simples-cego }\end{array}$ & $\begin{array}{l}\text { De acordo com os resultados, nenhuma diferença } \\
\text { significativa foi observada entre os dois grupos na } \\
\text { identificação de Pontos cefalométricos Ar ou Go } \\
\text { P> 0,05). No entanto, a taxa média de erro na } \\
\text { identificação do ponto Or foi significativamente } \\
\text { menor no grupo de smartphones. }\end{array}$ \\
\hline Abbasi, et al. 2020 . & $\begin{array}{l}\text { Pakistan Journal } \\
\text { of Medical } \\
\text { Sciences }\end{array}$ & $\begin{array}{l}\text { Determinar as percepções dos alunos } \\
\text { em relação ao e-learning durante o } \\
\text { bloqueio. }\end{array}$ & $\begin{array}{c}\text { Pesquisa } \\
\text { exploratória }\end{array}$ & $\begin{array}{l}\text { De acordo com os resultados gerais, } 77 \% \text { dos } \\
\text { alunos têm percepções negativas em relação ao } e \text { - } \\
\text { learning. } 76 \% \text { dos alunos usam dispositivos } \\
\text { móveis para o e-learning. }\end{array}$ \\
\hline
\end{tabular}

Fonte: Autores (2021).

Percebe-se que a utilização dos dispositivos móveis parece contribuir com a processo aprendizagem à partir da análise dos estudos selecionados, ainda que haja trabalhos nos quais os estudantes tenham percepções negativas sobre a aprendizagem por ferramentas tecnológicas, a maioria usa os dispositivos móveis em seu processo ensino-aprendizagem.

De acordo com o levantamento dos estudos, pode-se perceber que muitos alunos e professores já estão habituados a utilizar dispositivos móveis a fim de aprendizagem. Essa nova metodologia de ensino é denominada aprendizagem móvel, na qual as tecnologias móveis são inseridas em contexto educacional, de modo que a aprendizagem pode ser alcançada de forma síncrona e assíncrona, a qualquer momento e em qualquer lugar, com acesso instantâneo as informações (Suner, Yilmaz \& Piskin, 2019).

Essa realidade é possível, graças aos constantes avanços tecnológicos e a nova era da educação digital, onde uma maioria absoluta dos acadêmicos já possuem algum tipo de dispositivo com acesso à internet, tornando suas consultas facilitadas. Assim, para atender a essas necessidades, o uso de tecnologias de informação e comunicação (TIC) parece ser necessário (Kon, et al. 2016). 
De acordo com Rung, Warnke e Mattheos (2014) e Kon, et al. (2016) as principais ferramentas de educação e comunicação são aparelhos celulares, tablets e laptops. Dentre esses dispositivos podemos dizer que os mais populares para esse fim são Smartphones e os menos populares são os laptops. Uma série de aplicativos vem sendo utilizados para apresentação de casos clínicos da vida real para desenvolver a capacidade de pensamento crítico para estudantes de Odontologia (Deshpande, Chahande \& Rathi, 2017).

Assim, uma provável vantagem dos aplicativos móveis é que eles tornam as informações disponíveis o tempo todo, repetidamente, e podem ser acessadas facilmente e com melhor custo-benefício do que o texto para impressão. Ademais são portáteis, pequenos, sem fio e flexíveis, o que auxilia a utilização dessas ferramentas (Mergany, Dafalla \& Awooda, 2021).

A m-learning é um método adaptável, visto que permite ser executado de forma síncrona e assíncrona, de modo que ocorra um aprimoramento dos alunos, acerca de comunicação, e principalmente encorajando a uma aprendizagem autocentrada que permite uma maior comunicação e interação entre alunos e professores (Mergany, Dafalla \& Awooda, 2021).

Nos últimos anos os alunos vêm utilizando de tais ferramentas para uso pessoal, devido a ampliação do uso das mídias sociais e de sua facilidade de acesso, prejudicando o emprego delas para fins educacionais (Rung, Warnke \& Mattheos, 2014; Suner, Yilmaz \& Piskin, 2019). Além disso, alguns alunos relataram aspectos negativos como problemas associados ao carregamento de smartphones, distração por meio de aplicativos de mídia social e jogos, e problemas de conectividade à Internet (Rung, Warnke \& Mattheos, 2014; Mergany, Dafalla \& Awooda, 2021).

Estudos demonstram um resultado positivo sobre o uso das ferramentas digitais na percepção dos alunos, já que maioria deles considerou que o uso de dispositivos móveis e aplicativos de aprendizagem nos cursos de Odontologia era útil e que suas atitudes em relação a aprendizagem móvel eram positivas (Rung, Warnke \& Mattheos, 2014; Stamm, et al. 2019). Em concordância Mergany, Dafalla e Awooda (2021) indica que os alunos acreditam nas novas oportunidades de aprendizagem obtidas a partir dessa metodologia, contribuindo para a educação moderna em saúde, aumentando assim seu desempenho acadêmico.

Em divergência Abbasi, et al. (2020) demonstram que uma grande parcela dos alunos sentiu que a aprendizagem por dispositivos móveis tem pouco impacto na sua formação acadêmica e não estão preparados para o ensino eletrônico. A maioria dos alunos preferiu o ensino presencial em vez do e-learning. A tecnologia digital ainda não foi completamente adotada pelos estudantes de Odontologia para uso no ensino, visto que estão habituados com o ensino presencial e acreditam que exista limitações no que diz respeito aos aspectos práticos da aprendizagem no laboratório / ambiente clínico (Abbasi, et al. 2020).

Todavia, Golshah, Dehdar e Imani (2020) usou o aprendizado eletrônico como um complemento ao aprendizado tradicional e mostrou relativa superioridade em comparação com o aprendizado tradicional isolado. Assim, a aprendizagem móvel baseada em smartphone pode ser capaz de aprimorar o processo de aprendizagem, com alto potencial para promoção do conhecimento e incentivo aos alunos, especialmente quando usada em combinação com a aprendizagem tradicional.

Parece haver aceitabilidade por parte dos estudantes no que diz respeito à aprendizagem móvel e seu impacto positivo no processo ensino-aprendizagem, embora o ensino tradicional presencial ainda seja preferido. Frente a pandemia, da COVID19 (SARS-CoV-2) em que o distanciamento social é crucial para evitar a disseminação do vírus, as Instituições de Ensino foram obrigadas a migrar para o ensino online adotando novos métodos de ensino-aprendizagem, de modo que alunos e professores se comunicassem virtualmente. Diante dessa situação, a pandemia favoreceu e consolidou a expansão da aprendizagem ubíqua e móvel (Abbasi, et al. 2020). 


\section{Conclusão}

A partir do exposto percebe-se que a realidade imposta e acelerada pela pandemia da COVID-19, em relação ao uso das ferramentas digitais de ensino-aprendizagem, especialmente a aprendizagem móvel e ubíqua, é possível e já se mostra eficaz principalmente quando associada aos encontros presenciais. A manutenção do processo de ensino aprendizagem por meio de ferramentas digitais associado a recursos familiares aos estudantes pode se tornar uma grande potência no que diz respeito a amplificar a aquisição do conhecimento.

Mais estudos são necessários envolvendo os estudantes, docentes e Instituições de Ensino Superior e Cursos de Odontologia, no intuito de compreender na prática como a aprendizagem móvel e ubíqua se aplicam nas disciplinas de Odontologia.

\section{Referências}

Abbasi, S., Ayoob, T., Malik, A., \& Memon, S. I. (2020). Perceptions of students regarding e-learning during covid-19 at a private medical college. Pakistan journal of medical sciences., 36, 1-5.

Arce, J. R. \& Pegueros, J. P. C. (2017). Impacto del m-learning en el proceso de aprendizaje: habilidades y conocimiento. Revista iberoamericana para la investigacion y el desarrollo., 8(15), 363-386.

Ballesteros, V. A., Cardoso, O. I., Forero, S. L. \& Toledo, J. L. (2020). El aprendizaje móvil en educación superior: una experiencia desde la formación de ingenieros. Revista cientifica., 38, 243-257.

Bernardete, A. G. (2020). Possível reconfiguração dos modelos educacionais pós-pandemia. Instituto de estudos avançados da universidade de são Paulo., 34(100), 29-41.

Deshpande, S., Chahande, J. \& Rathi, A. (2017). A novel method to teach clinical decision making in prosthodontics. Education for health., $30(41)$, 1-4.

Dunleavy, G., Nikolaou, C. K., Nifakos, S., Rifat, A., Law, G. C. \& Car, L. T. (2019). Mobile digital education for health professions: systematic review and meta-analysis by the digital health education collaboration. Journal of medical internet research., 21(2), 225-245.

Elkheir, Z. \& Mutalib, A. A. (2015). Mobile learning applications desingning concepts and challenges: survey. Research jornal of applied sciences, engineering and technology.,10, 438-44.

Estrela, C. (2018). Metodologia científica: ciência, ensino, pesquisa. Editora artes médicas.

Golshah, A., Dehdar, F., Imani, M. M., \& Nikkerdar, N. (2020). Efficacy of smartphone-based mobile learning versus lecture-based learning for instruction of cephalometric landmark identification. Bmc medical education., 20(287),1-8.

Hinojo-Lucena, F. G., Inmaculada, A. D., Maria, P. C. R. \& José, M. R. R. (2019). Opinión de futuros equipos docentes de educación primaria sobre la implementación del mobile learning en el aula. Revista eletrônica Educare.,23(3), 283-299.

Kon, H., Kobayashi, H., Sakurai, N., Watanabe, K., Yamaga, Y. \& Ono, T. (2016). Personal computer versus personal computer/mobile device combination users' preclinical laboratory e-learning activity. Journal of investigative and clinical dentistry., 8(4), 1-8.

Leite, B. (2018). Aprendizagem Tecnológica Ativa. Revista Internacional de Educação Superior., 4(3), 580-609.

Medeiros, F. L. S. de, Araújo, M. C. de A., Almeida, A. B. C., Araújo Neto, A. P.., Santos, T. A., Feitosa, F. de S. Q., \& Costa, L. E. D. (2021). Impactos da pandemia da COVID 19 na educação odontológica: Visão de graduandos de Odontologia de uma instituição pública no Estado da Paraíba. Research, Society and Development, 10(7), e15310716089. https://doi.org/10.33448/rsd-v10i7.16089

Mergany, N. N., Dafalla, A. E. \& Awooda, E. (2021). Effect of mobile learning on academic achievement and attitude of Sudanese dental students: a preliminary study. BMC Medical Education., 21(121), 1-7.

Rung, A., Warnke, F. \& Mattheos, N. (2014). Investigating the use of smartphones for learning purposes by australian dental students. Jmir mhealth and uhealth., 2(2).

Santaella, M. L. (2019). A aprendizagem ubíqua substitui a educação formal? Revista de computação e tecnologia da puc-sp., 2 ,17-22.

Sales, A. B., \& Boscarioli, C. (2020). Uso de Tecnologias Digitais Sociais no Processo Colaborativo de Ensino e Aprendizagem. Revista Ibérica de Sistemas e tecnologias de informacao., 37, 82-98.

Silva, C. M., Aurea, T. M. T., Heloísa, G. C., Camila, A. B., Thaís, R. C. \& Pedro, I. C. A. S. (2021). Pandemia da COVID-19, ensino emergencial àdistância e Nursing Now: desafios à formação em enfermagem. Revista Gaúcha de Enfermagem., 42.

Stamm, T., Triller, I., Hohoff, A. \& Lubarsch, M. B. (2019). The tablet computer's impact on learning and national dental examination scores in orthodontics a mixed-method research. Head \& face medicine., 15(11), 1-8. 
Research, Society and Development, v. 10, n. 14, e88101421855, 2021

(CC BY 4.0) | ISSN 2525-3409 | DOI: http://dx.doi.org/10.33448/rsd-v10i14.21855

Suner, A., Yilmaz, Y. \& Kin, B. P. (2019). Mobile learning in dentistry: usage habits, attitudes and perceptions of undergraduate students. Peerj computer Science., 7.

Vieira, M. F. \& Silva, C. M. S. (2020). A educação no contexto da pandema de covid-19: uma revisão sistemática da literatura. Revista brasileira de informática na educação., 28, 1013-1031. 